\title{
Uncoupler Resistance in Escherichia coli: the Role of Cellular Respiration
}

\author{
By PHILIP G. QUIRK, ${ }^{1 *}+$ MICHAEL R. JONES,${ }^{2} \ddagger$ \\ ROBERT S. HAWORTH, ${ }^{2}$ R. BRIAN BEECHEY ${ }^{2}$ \\ AND IAIN D. CAMPBELL' \\ ${ }^{1}$ Department of Biochemistry, University of Oxford, South Parks Road, Oxford OXI 3QU, UK \\ ${ }^{2}$ Department of Biochemistry and Agricultural Biochemistry, University College of Wales, \\ Aberystwyth, Dyfed SY23 3DD, UK
}

(Received 8 March 1989; revised 12 June 1989; accepted 10 July 1989)

\begin{abstract}
Bioenergetic properties of a mutant strain of Escherichia coli $\mathrm{K} 12$ designated TUV, which is resistant to the protonophoric uncoupling agent 4,5,6,7-tetrachloro-2-trifluoromethylbenzimidazole (TTFB) have been compared with those of its non-resistant parent, $E$. coli K12 Doc-S. Strain TUV grew and respired some $20-30 \%$ faster than strain Doc-S, and was cross-resistant to carbonylcyanide $p$-(trifluoromethoxy)phenylhydrazone and triphenyltin, but not to 2,4dinitrophenol. Phosphorus nuclear magnetic resonance demonstrated the TTFB-mediated collapse of the transmembrane $\mathrm{pH}$ gradient at identical rates in starved cells of both strains, indicating that uncoupler access and function were unimpaired in the mutant under these conditions. Strain TUV displayed enhanced uncoupler resistance and maintained intracellular pH and ATP levels only when respiring. On the other hand, strain TUV also showed increased resistance to novobiocin, implying that its outer wall permeability had been lowered. We suggest that the active resistance of strain TUV results from the exclusion of uncoupler by the interaction of inner and outer membrane components in a manner modulated by the degree of cellular energization.
\end{abstract}

\section{INTRODUCTION}

It is generally agreed that a transmembrane proton electrochemical gradient plays an indispensable role in cellular energy transduction, linking the processes of respiration, ATP synthesis and active transport. One attractive feature of this chemiosmotic theory (Mitchell, 1961 ) is its ability to explain the mechanism of action of uncoupling agents, which are proposed to act as protonophores and dissipate the transmembrane protonmotive force $(\Delta p)$. Excellent correlations have been observed between uncoupler potency in vivo and protonophoric activity in vitro (McLaughlin \& Dilger, 1980), but several uncouplers have been reported to bind with high affinity to integral membrane proteins of both bacteria and mitochondria (Katre \& Wilson, 1980; Partis et al., 1984). It has been suggested that such interactions are important in uncoupling in vivo, and that uncoupler resistance arises from mutations affecting these binding proteins (Kell, 1982). The elucidation of the molecular mechanism of such resistance is thus important, both for our understanding of uncoupler action and for the information it may provide concerning the mechanism of cellular energy coupling.

$\dagger$ Present address: Mount Sinai Medical Center, Department of Biochemistry, Box 1020, 1 Gustave L. Levy Place, New York, NY 10029, USA.

‡ Present address: Department of Biochemistry, University of Birmingham, PO Box 363, Birmingham B15 2TT, UK.

Abbreviations: CCCP, carbonylcyanide $m$-chlorophenylhydrazone; DNP, 2,4-dinitrophenol; FCCP, carbonylcyanide $p$-(trifluoromethoxy)phenylhydrazone; MDP, methylenediphosphonic acid; MeP, methylphosphonate; TPP ${ }^{+}$, tetraphenylphosphonium; TPT, triphenyltin; TTFB, 4,5,6,7-tetrachloro-2trifluoromethylbenzimidazole. 
Jones \& Beechey (1987) isolated two mutant derivatives of Escherichia coli K12 Doc-S, designated strains TUV and CUV, which were resistant to the uncouplers 4,5,6,7-tetrachloro2-trifluoromethylbenzimidazole (TTFB) and carbonylcyanide $m$-chlorophenylhydrazone (CCCP). An enhanced resistance of growth, oxidative phosphorylation and proline transport was demonstrated. The parent strain, Doc-S, possesses a defective outer wall, which allows bulky hydrophobic species, such as deoxycholate, to penetrate to the cell membrane (Ahmed \& Booth, 1983). Deoxycholate sensitivity was retained by strains TUV and CUV, and uncouplers were therefore believed to have equal access to their sites of action in all three strains, although this could not be proved directly.

Phosphorus nuclear magnetic resonance spectroscopy ( $\left.{ }^{31} \mathrm{P}-\mathrm{NMR}\right)$ provides an attractive means of investigating these cells. It allows the simultaneous non-invasive monitoring of many metabolically important compounds (inorganic phosphate, nucleotides, glycolytic intermediates), and the rapid estimation of intra- and extracellular $\mathrm{pH}$ from the corresponding phosphate chemical shifts. ${ }^{31} \mathrm{P}-\mathrm{NMR}$ spectroscopy has been used extensively in studies of $E$. coli bioenergetics (e.g. Ogawa et al., 1978; Ugurbil et al., 1978; Slonczewski et al., 1981), but has not been applied previously to the problem of uncoupler resistance.

In this paper, we extend our earlier work (Jones et al., 1986; Jones \& Beechey, 1987), making particular use of NMR spectroscopy to address the question of whether TTFB functions with equal efficacy in strains Doc-S and TUV. We present evidence indicating the active, energyrequiring nature of uncoupler resistance in strain TUV. Other data, however, suggest that some change in cell wall permeability is involved, a mutation which might be expected to produce passive uncoupler resistance (i.e. unaffected by the degree of cell energization). A possible scheme for rationalizing these apparently contradictory findings is proposed.

\section{METHODS}

Materials. Carbonylcyanide $p$-(trifluoromethoxy)phenylhydrazone (FCCP) and triphenyltin (TPT) were purchased from Aldrich; methylphosphonate (MeP) from Alfa Produkte, Karlsruhe; 2,4-dinitrophenol (DNP) from BDH; and ${ }^{2} \mathrm{H}_{2} \mathrm{O}$, amino acids, buffers, methylenediphosphonic acid (MDP) and novobiocin from Sigma. All chemicals were of at least analytical grade.

Organisms. E. coli $\mathrm{K} 12$ Doc-S (laci- $z^{+} y^{+} a^{+}$pro trp his met), and its uncoupler-resistant derivative strains TUV and CUV have been described previously (Ahmed \& Booth, 1983; Jones \& Beechey, 1987). E. coli strains MRE600 and J53 RP4, both with wild-type cell walls, were also used.

Growth media. LB medium and succinate minimal medium were as given previously (Jones \& Beechey, 1987). Glycerol minimal medium contained $5 \mathrm{ml}$ glycerol $\mathrm{l}^{-1}$, replacing succinate. Where appropriate, uncouplers were added aseptically, immediately before inoculation with cells.

Suspension media. These gave some osmolarity and buffering capacity but lacked respiratory substrates, which were added separately. Three media were employed, designed to provide optimal buffering capacity at different $\mathrm{pH}$. All contained $40 \mathrm{mM}-\mathrm{NaCl}$ and $10 \mathrm{mM}-\mathrm{KCl}$, supplemented by either $2-(N$-morpholino)ethanesulphonic acid (MES; $150 \mathrm{mM}$ ), pH 6; $N$-(2-acetamido)iminodiacetic acid (ADA; $100 \mathrm{mM}$ ) plus piperazine- $N$, $N^{\prime}$-bis-(2ethanesulphonic acid) (PIPES; $40 \mathrm{mM}$ ), pH 6.1; or 3-( $N$-morpholino)propanesulphonic acid (MOPS; $150 \mathrm{mM}$ ), $\mathrm{pH}$ 7. These solutions are designated MES, ADA/PIPES and MOPS medium, respectively.

Growth. Cells were maintained and grown essentially as before (Jones \& Beechey, 1987), except that growth was at $37^{\circ} \mathrm{C}$ and was monitored spectrophotometrically at $600 \mathrm{~nm}$.

Harvesting. Cells were harvested at mid-exponential phase $\left(\mathrm{OD}_{600} 0.8-1 \cdot 2\right)$ by centrifugation at $17700 \mathrm{~g}$ for $10 \mathrm{~min}$ at $4{ }^{\circ} \mathrm{C}$. They were washed once with the appropriate suspension medium, resuspended to $40-50 \mathrm{mg}$ protein $\mathrm{ml}^{-1}$, kept on ice and used within $14 \mathrm{~h}$.

Assays. Protein was assayed by the Lowry method, using bovine serum albumin as standard. Cell samples were first lysed by vigorous mixing with an equal volume of $2 \mathrm{M}-\mathrm{NaOH}$.

TTFB was assayed spectrophotometrically. Its absorption coefficient at $301 \mathrm{~nm}$ in water was measured as $6530 \mathrm{M}^{-1} \mathrm{~cm}^{-1}$.

The binding of TTFB to cells was assayed under conditions comparable to those of an NMR experiment: MOPS medium ( $\mathrm{pH} 7$ ), $25 \mathrm{mg}$ protein $\mathrm{ml}^{-1}$. Samples were incubated anaerobically for $10 \mathrm{~min}$ with TTFB (25$500 \mu \mathrm{M}$ ), then centrifuged for $1 \mathrm{~min}$ (Eppendorf 5412 microfuge). The supernatant was centrifuged again to ensure complete removal of cells, diluted $1: 2$ with water and its $A_{301}$ determined, reading against a control sample to which only ethanol had been added. TTFB binding was calculated from the difference between the amount remaining in the supernatant and that originally added. To avoid introducing errors from Scatchard linearization, 
$K_{\mathrm{d}}$ was estimated directly from the binding curve data, using the Marquardt algorithm for non-linear regression (Duggleby, 1984).

The procedure for monitoring deoxycholate sensitivity has been described previously (Jones \& Beechey, 1987); tests of novobiocin sensitivity were conducted in an identical manner.

Respiratory studies. Harvested cells, grown in the absence of uncoupler, were studied using a Clark-type oxygen electrode (Yellow Springs YSI 5331) inserted into a water-jacketed, $3.3 \mathrm{ml}$ reaction chamber, maintained at $25^{\circ} \mathrm{C}$.

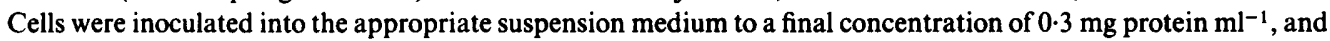
reoxygenated when necessary by bubbling for short periods with $\mathrm{O}_{2} / \mathrm{CO}_{2}(95: 5, \mathrm{v} / \mathrm{v})$.

Membrane potential. Membrane potential $(\Delta \psi)$ was measured according to the method of Ahmed \& Booth (1983), using $\left[{ }^{14} \mathrm{C}\right]$ tetraphenylphosphonium (TPP+).

NMR studies. The spectrometer was constructed around an Oxford Instruments 8.5 T superconducting widebore magnet, interfaced to Nicolet computing equipment. Standard samples [15 $\mathrm{ml}$ bacterial suspension, $1 \mathrm{ml}$ ${ }^{2} \mathrm{H}_{2} \mathrm{O}$ (field/frequency lock), $0.2 \mathrm{ml}$ silicone antifoam, $0.2 \mathrm{ml} 200 \mathrm{mM}-\mathrm{MeP}$ (internal reference) and $0.1 \mathrm{ml} 100 \mathrm{mM}$ $\mathrm{NaH}_{2} \mathrm{PO}_{4}$; final cell concentration $25 \mathrm{mg}$ protein $\mathrm{ml}^{-1}$ ] were placed in a $20 \mathrm{~mm}$ diameter, flat-bottomed $\mathrm{NMR}$ tube, and maintained at $21^{\circ} \mathrm{C}$. A capillary containing MDP served as an external reference. The antifoam was tested for uncoupling activity, but showed none.

Samples were oxygenated continuously by two flows of $\mathrm{O}_{2} / \mathrm{CO}_{2}(95: 5, \mathrm{v} / \mathrm{v}):$ a flow of $130 \mathrm{ml} \mathrm{min}^{-1}$ through four capillaries inserted to the bottom of the sample, and a surface-directed flow of $11 \mathrm{~min}^{-1}$ (Slonczewski et al., 1981). In a bench test of a sample of strain Doc-S respiring on succinate, an oxygen tension of $250 \mu \mathrm{M}-\mathrm{O}_{2}$ was maintained, well above the $K_{\mathrm{m}}$ of $E$. coli MRE600 for $\mathrm{O}_{2}(0.7 \mu \mathrm{M})$ (Ogawa et al., 1978).

Fourier transform ${ }^{31} \mathrm{P}-\mathrm{NMR}$ spectra were acquired at $145.8 \mathrm{MHz}$ with broad-band proton decoupling, and using a single $40^{\circ}$ pulse in order to allow a rapid recycle time (405 ms, including a relaxation delay of $\left.200 \mathrm{~ms}\right)$. Each transient comprised 4096 data points and the sweep width was $\pm 5000 \mathrm{~Hz}$. Spectra were accumulated from blocks of between 128 and 1480 transients (total acquisition times 0.9-10.4 min). A 10 s delay was programmed between the acquisition of each spectrum, during which time substrates and reagents could be added via an injection line. Line-broadening of $20 \mathrm{~Hz}$ was imposed on the transformed spectra, which were assigned from the literature (Ugurbil et al., 1978; Slonczewski et al., 1981).

Titration curves of chemical shift against $\mathrm{pH}$ were constructed for phosphate and $\mathrm{MeP}$ by treating samples with small volumes of $1 \mathrm{M}-\mathrm{NaOH}$ and $1 \mathrm{M}-\mathrm{HCl}$; the compounds behaved identically in all three suspension media. The chemical shifts of intracellular phosphate and MeP were assumed to follow the same curves as in the media. This assumption was verified directly for $\mathrm{pH}$ values above 6.3 by titrating samples of anaerobic $E$. coli Doc-S following 40 min incubation with $200 \mu \mathrm{M}$-TTFB. Below pH 6.3, two phosphate resonances were resolved, probably due to the presence of a residual transmembrane $\mathrm{pH}$ gradient, opposing a Donnan membrane potential (Ogawa et al., 1981 ). Such a $\Delta \mathrm{pH}$ (around $0.2 \mathrm{pH}$ units) would not be competent to drive ATP synthesis, as there would be no net protonmotive force.

\section{RESULTS}

\section{Binding of TTFB to cells}

The binding properties of TTFB appeared virtually identical in strains Doc-S, TUV and MRE600, being monophasic with a $K_{d}$ in strain TUV of $217 \pm 3 \mu \mathrm{M}$ (two determinations). This probably represents non-specific binding, as small amounts of tight, specific binding $\left(K_{\mathrm{d}}<10 \mu \mathrm{M}\right.$; e.g. Partis et al., 1984) would be impossible to detect with this assay. Under typical conditions employed for NMR studies ( $100 \mu \mathrm{M}$-TTFB), around $75 \%$ of the uncoupler is bound to the cells.

\section{Growth and cross-resistance properties}

At $37^{\circ} \mathrm{C}$ in the absence of uncoupler, the growth rate of strain TUV on succinate minimal medium, $\mathrm{pH} 7$, was up to $25 \%$ higher than that of strain Doc-S (fastest doubling times: TUV, $90 \mathrm{~min}$; Doc-S, $120 \mathrm{~min}$ ), consistent with previous observations at $30^{\circ} \mathrm{C}$ (Jones \& Beechey, 1987). Under these conditions, the respiratory rate of strain TUV was $17 \pm 5 \%$ greater than that of strain Doc-S (three separate determinations on cultures growing simultaneously in the same incubator), although the actual rates varied somewhat [strain Doc-S, $800 \mathrm{ng}$-atom O min ${ }^{-1}$ (mg protein $)^{-1}, \pm 10 \%$.

The results of a series of growth studies are summarized in Table 1. Compared with strain Doc-S, strain TUV displayed markedly increased resistance to TTFB, FCCP and TPT, but was not cross-resistant to DNP. Strain CUV was three times more resistant than strain TUV to 
Table 1. Uncoupler-resistant growth of E. coli strains

Figures indicate the concentration $(\mu \mathrm{M})$ of uncoupler causing a $50 \%$ reduction in growth rate, relative to control flasks, under the specified conditions. The same amount of ethanol (solvent for the uncouplers) was present in all flasks and had negligible effects on growth. ND, Not determined.

\begin{tabular}{lclrrrrr}
$\begin{array}{l}\text { Carbon } \\
\text { source }\end{array}$ & $\mathrm{pH}$ & Uncoupler & Strain... & \multicolumn{4}{c}{ Uncoupler $I_{50}(\mu \mathrm{M})$} \\
Succinate & 7 & TTFB & & TUV & CUV & MRE600 \\
Succinate & 6 & TTFB & 12 & 120 & 120 & 25 \\
Glycerol & 7 & TTFB & 3 & 35 & 35 & ND \\
Succinate & 7 & FCCP & 10 & 150 & ND & ND \\
Succinate & 7 & DNP & 2 & 25 & 80 & $<5$ \\
Succinate & 7 & TPT & 80 & 70 & 150 & 150 \\
& & & $<0.5$ & 4 & 3 & ND
\end{tabular}

FCCP, consistent with results obtained with the structurally similar CCCP (Jones \& Beechey, 1987), and was weakly cross-resistant to DNP, but its resistance to TTFB and TPT was not further enhanced. Resistance to TTFB did not require any specific carbon source, that of strain TUV being unaffected by the substitution of glycerol (Table 1) or acetate (Jones \& Beechey, $1987)$ for succinate. These respiratory substrates are all non-fermentable. In the three strains tested, TTFB was three to four times more potent at $\mathrm{pH} 6$ than at $\mathrm{pH} 7$. The TTFB-induced conductance of phospholipid bilayers shows a similar $\mathrm{pH}$ dependence, being 3.5 times higher at pH 6 than at pH 7 (Dilger \& McLaughlin, 1979).

TPT may uncouple cells by catalysing transmembrane $\mathrm{Cl}^{-} / \mathrm{OH}^{-}$exchange (Selwyn et al., 1970), but can also inhibit the $F_{0} F_{1}$ ATP synthase directly (Kagawa et al., 1979). The relative importance of these processes is unclear, but tributyltin requires at least $40 \mathrm{~mm}$ extracellular $\mathrm{Cl}^{-}$ to uncouple $E$. coli effectively (Sedgwick $e t$ al., 1984). It thus seems unlikely that TPT would uncouple cells in succinate minimal medium, containing less than $2 \mathrm{mM} \mathrm{Cl}^{-}$(from histidine. $\mathrm{HCl}$ ), and more likely that the effects of the compound arise from inhibition of the ATP synthase.

\section{Sensitivity to novobiocin}

In order to compare the cell wall permeabilities of the strains, their sensitivity to novobiocin, an inhibitor of DNA gyrase (Cozzarelli, 1980), was investigated (Fig. 1). Strain Doc-S was clearly the most sensitive, while the resistance of strains TUV and CUV appeared similar to that of the wild-type J53 RP4.

\section{Deprivation of respiratory substrate}

Strain TUV was grown overnight in LB medium, then transferred to incomplete minimal medium, $\mathrm{pH} 7$, containing $100 \mu \mathrm{M}$-TTFB but lacking both succinate and citrate. After various preincubation periods, succinate and citrate were provided and subsequent growth was monitored. The rationale was that only active, energy-requiring resistance would be impaired by this deprivation of respiratory substrate in the presence of uncoupler, with passive resistance remaining unaffected.

In control experiments done in the absence of uncoupler, the lag time of strain TUV following the reintroduction of the carbon sources was under $30 \mathrm{~min}$, even after $120 \mathrm{~min}$ preincubation in incomplete medium. Preincubation for up to $5 \mathrm{~min}$ in incomplete medium containing $100 \mu \mathrm{M}$ TTFB did not affect this lag time, but 10 min preincubation increased it to $10-12 \mathrm{~h}$ (three experiments). Similar results were obtained when FCCP $(15 \mu \mathrm{M})$ was substituted for TTFB.

\section{Respiratory studies}

The results of a typical experiment comparing harvested cells of strains Doc-S and TUV are shown in Fig. 2. Cells were introduced into the reaction chamber and incubated in the absence of respiratory substrate or uncoupler. Following an initial short burst of respiration, oxygen consumption in both strains fell to a constant low rate. After 15 min incubation, $100 \mu \mathrm{M}$-TTFB 


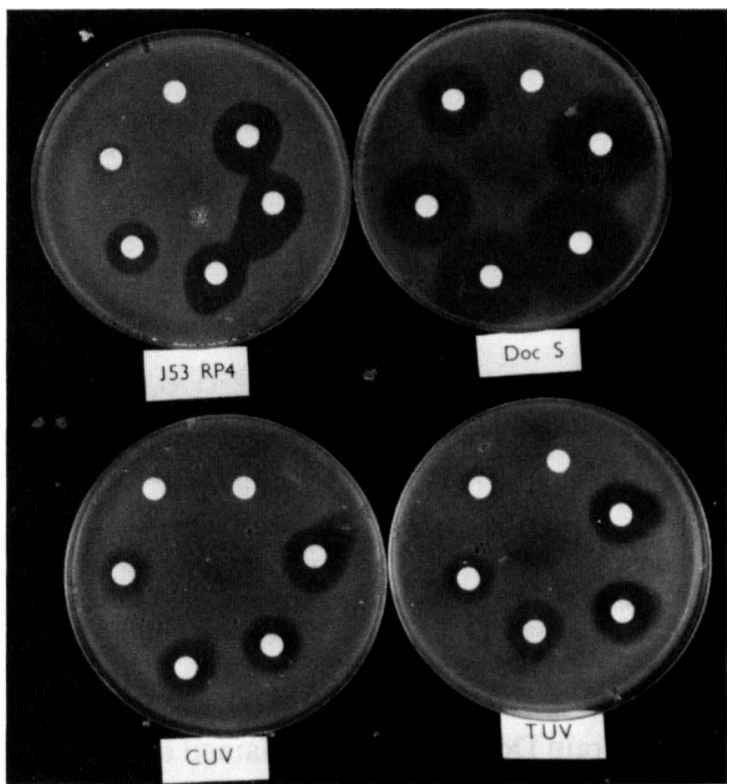

Fig. 1. Sensitivity of $E$. coli strains to novobiocin. The filter discs on each plate were treated with (reading anticlockwise from the top) $0,35,180,350,530$ and $700 \mu \mathrm{g}$ novobiocin, respectively. The enhanced resistance of strains TUV and CUV, compared with strain Doc-S, is readily apparent.

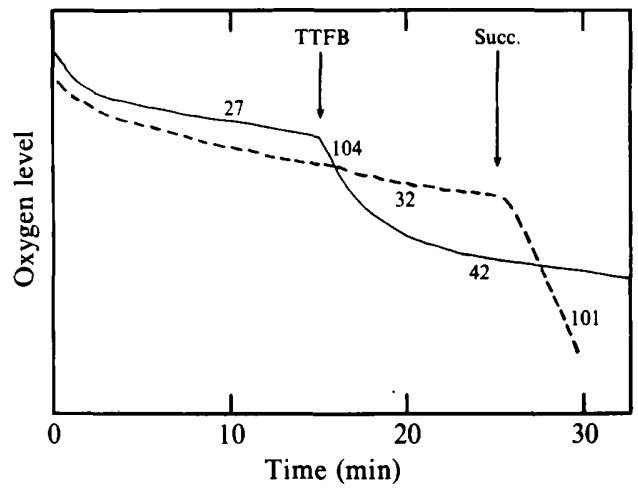

Fig. 2. Respiratory properties of $E$. coli Doc-S and TUV. Cells were introduced into the reaction chamber (containing MOPS medium) at time zero. TTFB $(100 \mu \mathrm{M})$ was added after $15 \mathrm{~min}$, and succinate $(5 \mathrm{mM})$ after a further $10 \mathrm{~min}$. Respiratory rates [ng-atom $\mathrm{O} \mathrm{min}^{-1}\left(\mathrm{mg}\right.$ protein $\left.{ }^{-1}\right)$ ] are indicated on the curves. - , Doc-S; ---, TUV.

was added, a concentration which prevents the growth of strain Doc-S, but not that of strain TUV (Table 1). With strain Doc-S, a further burst of respiration ensued, believed to represent the rapid oxidation of endogenous substrate reserves. A similar loss of respiratory control has been noted in another strain of $E$. coli K12 (Burstein et al., 1979). The respiratory rate of strain Doc-S gradually declined towards its previous level, but the addition of succinate $10 \mathrm{~min}$ later failed to elicit any respiratory response. Respiration of strain TUV, in contrast, was little affected by $10 \mathrm{~min}$ incubation with TTFB and cells continued to respond to added succinate, even though similar incubations with uncoupler caused large increases in culture lag times (see previous section). The reason for this discrepancy is unknown. 


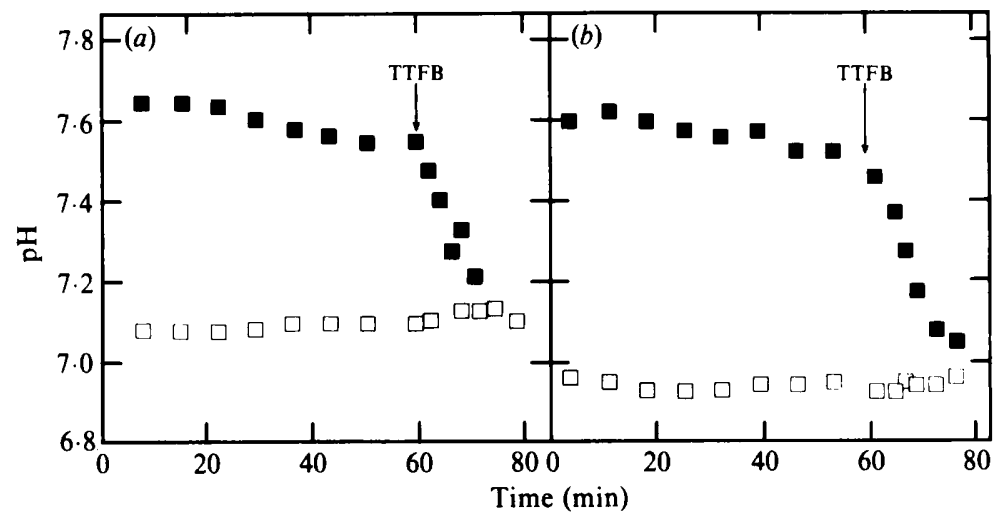

Fig. 3. Effects of TTFB on $\triangle \mathrm{pH}$ of starved cells of $E$. coli Doc-S and TUV. Results are representative of several experiments (Doc-S, $n=2$; TUV, $n=3$ ). $\square$, Intracellular $\mathrm{pH} ; \square$, extracellular pH. (a) Sample: $16.5 \mathrm{ml}$ strain Doc-S, MOPS medium. Oxygenation commenced at time zero; TTFB (100 $\mu \mathrm{M})$ was added 60 min later. (b) Identical sample and protocol to $(a)$, but using strain TUV.

These effects of TTFB on strain Doc-S could be explained in two ways: uncoupler-binding to membrane proteins causing a direct inhibition of succinate transport and/or respiration; or the dissipation of $\Delta p$ removing the driving force for transport (Gutowski \& Rosenberg, 1975). The first alternative is considered unlikely. If metabolite transport were inhibited directly in strain Doc-S, then the transporters for succinate, glycerol and acetate must all have become resistant in strain TUV, since TTFB-resistant growth occurs on all three substrates. Furthermore, succinate respiration by strain Doc-S was not inhibited immediately upon addition of TTFB, but continued unaffected for a further $60 \mathrm{~min}$ (data not shown), presumably the time required for the oxidation of all endogenous substrate. Thus TTFB does not inhibit respiration or transport directly, and strain TUV must either maintain $\Delta p$ in the presence of TTFB or transport succinate at values of $\Delta p$ below those required by strain Doc-S.

\section{Membrane potential}

A membrane potential of $130 \mathrm{mV}$ was measured with strain Doc-S suspended at $\mathrm{pH} 7$, in good agreement with a previous study (Ahmed \& Booth, 1983). In contrast, strain TUV took up the probe only after pretreatment with EDTA, when a $\Delta \psi$ of $135 \mathrm{mV}$ was obtained. Addition of TTFB [ $4 \mathrm{nmol}$ (mg dry weight $)^{-1}$ ] caused complete dissipation of $\Delta \psi$ in both strains. These data provide further evidence for a change in envelope permeability in strain TUV.

\section{${ }^{31} P-N M R$ spectroscopy}

Effects of TTFB on cells lacking respiratory substrate. Cell samples were oxygenated in situ for $60 \mathrm{~min}$ in the absence of added substrate in order to deplete them of endogenous reserves. During this starvation the $\beta$-NTP resonance declined by up to $80 \%$. The starved cells were then treated with TTFB and subsequent changes in cytoplasmic and extracellular pH were monitored. TTFB dissipated $\Delta \mathrm{pH}$ equally rapidly in starved cells of both strains (Fig. 3).

Effects of TTFB on succinate-fed cells. Cell samples (in MES or ADA/PIPES medium) were incubated with succinate, then challenged with TTFB. Preliminary work, done in the absence of uncoupler, revealed the expected extracellular alkalinization as succinate was transported into the cells via the succinate $/ \mathrm{H}^{+}$symport (Gutowski \& Rosenberg, 1975), but intracellular $\mathrm{pH}$ was maintained at between 7.3 and 7.5 . In these experiments, $\Delta \mathrm{pH}$ could be reduced by both succinate transport and uncoupler action, but the two processes were distinguishable by the direction of net proton movement: succinate uptake causes extracellular alkalinization, intracellular $\mathrm{pH}$ remaining constant, whereas uncoupling is associated with intracellular acidification, extracellular $\mathrm{pH}$ remaining constant. 

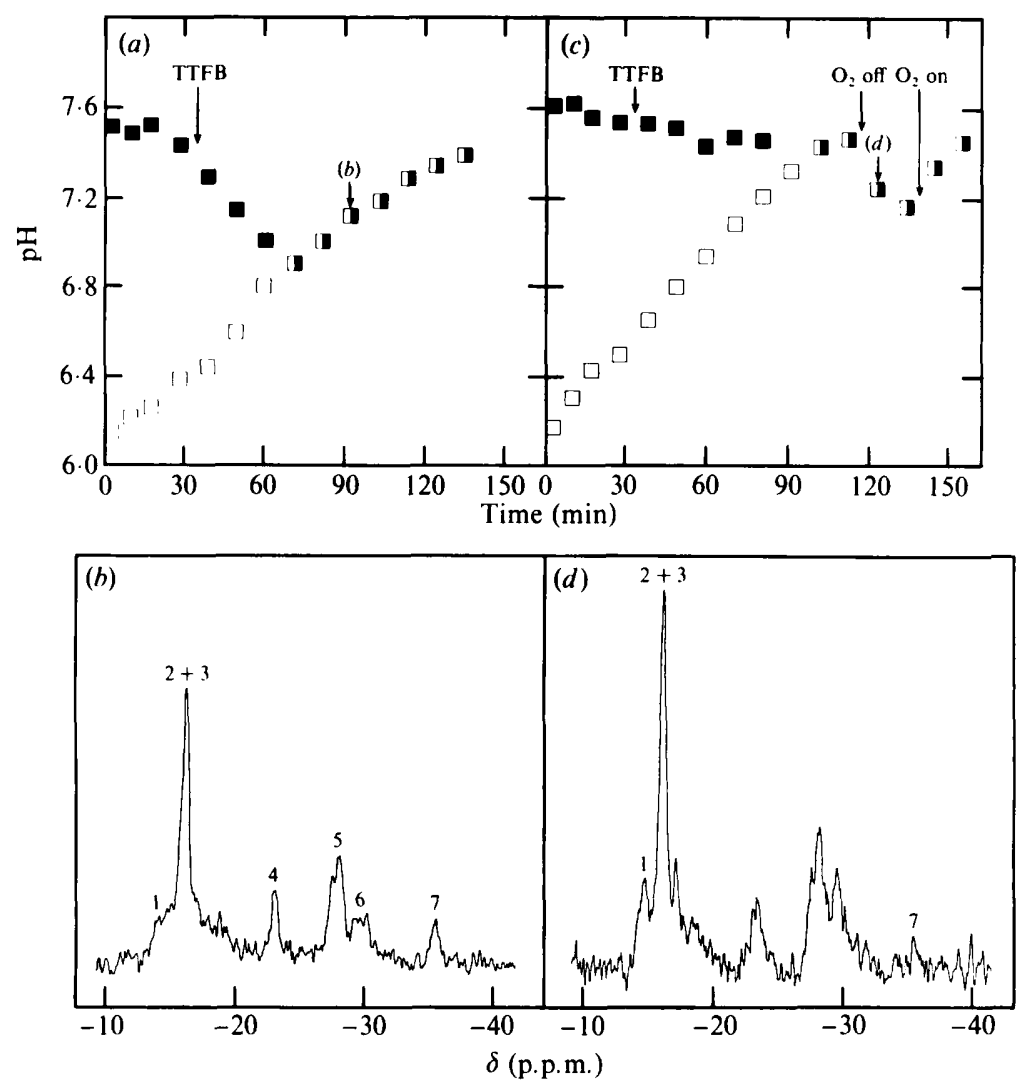

Fig. 4. Effects of TTFB on $\triangle \mathrm{pH}$ and intracellular ATP of cells respiring on succinate. Results are representative of several experiments (Doc-S, $n=3$; TUV, $n=4$ ). $\square$, Intracellular pH; $\square$, extracellular pH. (a) Sample: $12.8 \mathrm{ml}$ strain Doc-S, ADA/PIPES medium. Oxygenation commenced at time zero, with succinate $(78 \mathrm{mM})$ present; TTFB $(150 \mu \mathrm{M})$ was added 33 min later. (b) ${ }^{31} \mathrm{P}$-NMR spectrum $(1480$ transients) of strain Doc-S acquired 87.0-98.6 min after time zero, as indicated in (a). Assignments: 1 , phosphomonoesters; 2 , intracellular $\mathrm{P}_{i} ; 3$, extracellular $\mathbf{P}_{\mathrm{i}} ; 4, \gamma$-NTP $+\beta$-NDP; $5, \alpha$-NTP + $\alpha$-NDP + NAD $(P)(H) ; 6$, NDP-glucose; 7, $\beta$-NTP. Sample and initial treatment identical to $(a)$, but using strain TUV. Oxygenation interrupted after $118 \mathrm{~min}$ and more succinate (72 mM) added. Oxygenation resumed 21 min later. (d) Spectrum (1480 transients) of strain TUV acquired $118 \cdot 8-129.4 \mathrm{~min}$ after time zero, as indicated in $(c)$, and plotted with a vertical scale expansion twice that of $(b)$.

The addition of $150 \mu \mathrm{M}$-TTFB to strain Doc-S (Fig. $4 a$ ) caused a gradual intracellular acidification, although succinate uptake continued and the medium continued to alkalinize. Intracellular acidification was significantly slower than that observed with starved cells (Fig. $3 a$ ). After the dissipation of $\Delta \mathrm{pH}$ was complete, the indicated $\mathrm{pH}$ was $6.95 \pm 0 \cdot 1$ in all three experiments. A slow alkalinization followed, but the phosphate signal did not split, indicating that intra- and extracellular $\mathrm{pH}$ rose together. A similar pattern of events occurred with strain TUV (Fig. $4 c$ ) but intracellular $\mathrm{pH}$ in all four cases was maintained at $7 \cdot 4 \pm 0 \cdot 1$, despite the presence of the uncoupler.

Even after the dissipation of $\Delta \mathrm{pH}$, both strains retained a large $\beta$-NTP resonance (Fig. $4 b$ ). Interruption of the oxygen supply caused a rapid decline in this resonance (Fig. $4 d$ ), indicating that oxidative phosphorylation had continued to operate while oxygen was available. The availability of substrate thus allows even strain Doc-S to resist uncoupling to some extent. Higher concentrations of TTFB $(200-750 \mu \mathrm{M})$ caused significant intracellular acidification and the disappearance of the $\beta$-NTP resonance in both strains (data not shown). 

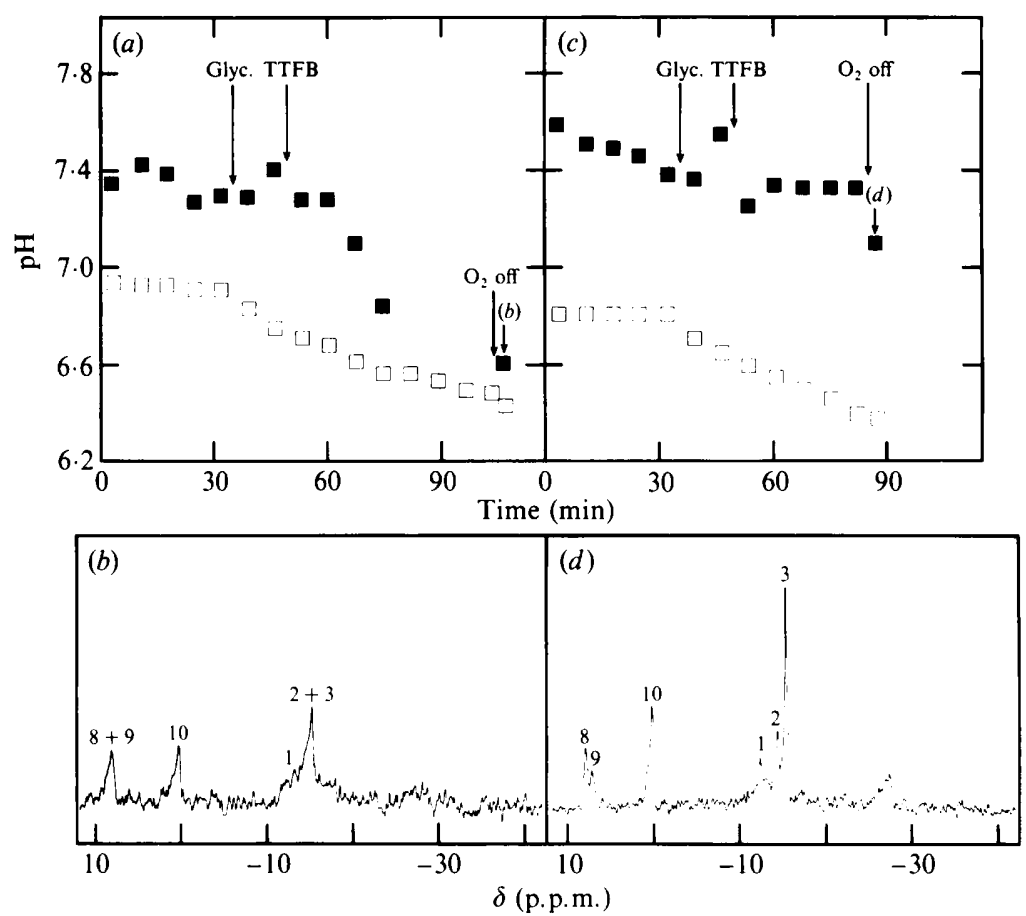

Fig. 5. Effects of TTFB on $\triangle \mathrm{pH}$ and intracellular ATP of cells respiring on glycerol. Results are representative of several experiments (Doc-S, $n=2$; TUV, $n=5$ ). $\square$, Intracellular $\mathrm{pH}$; $\square$, extracellular pH. Assignments as for Fig. 4, plus: 8, extracellular methylphosphonate (MeP); 9, intracellular MeP; 10, methylenediphosphonic acid (MDP). (a) Sample: 16.5 ml strain Doc-S, MOPS medium. Oxygenation commenced at time zero. Glycerol $(79 \mathrm{~mm})$ was added after $46 \mathrm{~min}$, followed $14 \mathrm{~min}$ later by TTFB $(100 \mu \mathrm{M})$. (b) Spectrum ( 512 transients) acquired 105.3-108.9 min after time zero, as indicated in $(a)$. Oxygenation was interrupted during this period, in order to improve the signal/noise ratio. (c) Identical protocol to $(a)$, but using strain TUV. (d) Spectrum (512 transients) acquired without oxygenation $105 \cdot 3-108.9 \mathrm{~min}$ after time zero, as indicated in $(c)$.

Effects of TTFB on glycerol-fed cells. Cells were grown in glycerol minimal medium ( $\mathrm{pH} 7$ ), harvested and resuspended in MOPS medium. In control experiments without uncoupler, glycerol metabolism, presumably phosphorylation, caused the depletion of intracellular NTP and phosphate (which sometimes became difficult to detect), and a corresponding increase in the phosphomonoester resonance. Extracellular $\mathrm{pH}$ gradually fell, possibly due to the excretion of acidic products of incomplete glycerol oxidation. Both strains accumulated MeP, as shown by the splitting of its NMR resonance (Slonczewski et al., 1981).

The addition of TTFB to strain Doc-S (Fig. $5 a$ ) initiated an intracellular acidification, and $\Delta \mathrm{pH}$ fell, even though acidification of the medium continued. In contrast, strain TUV (Fig. $5 c$ ) maintained its intracellular $\mathrm{pH}$ above $7 \cdot 3$, and $\Delta \mathrm{pH}$ gradually increased. The continued resolution of intra- and extracellular phosphate and $\mathrm{MeP}$ resonances illustrates the maintenance of a $\Delta \mathrm{pH}$ by strain TUV, but not by strain Doc-S (Fig. $5 b, d)$. At a higher concentration of TTFB $(200 \mu \mathrm{M}), \Delta \mathrm{pH}$ collapsed rapidly in both strains (data not shown).

\section{DISCUSSION}

Uncoupler resistance is a complex phenomenon, with resistant cell lines often displaying many phenotypic alterations. In order to better comprehend the nature of resistance in $E$. coli TUV and CUV, we have drawn a distinction between active and passive mechanisms, and attempted to establish their relative importance in vivo. This discussion will focus mainly on the better characterized strain TUV. 
Previous studies of uncoupler resistant $E$. coli have provided some indications of active resistance. In the first-reported strain, SWL14 (Date et al., 1980), proline transport was resistant in both cells and right-side-out vesicles. An enhanced resistance to uncoupler-mediated dissipation of $\Delta \mathrm{pH}$ was reported for vesicles of a further strain, CM22 (Ito et al., 1983). In E. coli UV6, the best-characterized mutant to date, $\Delta \mathrm{pH}$ of whole cells was also reported as being less susceptible to collapse by uncoupler, but vesicles were not examined (Sedgwick et al., 1984). Unfortunately, interpretation of results with this strain was complicated by the occurrence of illdefined $\mathrm{H}^{+}$movements, possibly across the outer membrane; it was hoped that the use of derivatives of strain Doc-S would avoid such problems. Strains CM22 and UV6 both showed multiple cross-resistances, similar to those of strains TUV and CUV.

Two lines of evidence indicate the importance of active resistance in strain TUV. Firstly, the large increases in lag times when uncoupler-treated cultures are temporarily deprived of substrate point to some protective effect of respiratory metabolism. Secondly, NMR samples of strain TUV show superior resistance only when respiratory substrate is available. The ability of strain Doc-S to maintain intracellular NTP in the presence of TTFB is also enhanced by the presence of substrate, provided that the intracellular $\mathrm{pH}$ has not fallen too low. The role of respiratory substrate in uncoupler resistance appears not to have been examined previously in this manner, but has proved informative in this study.

The immediate, uncoupler-mediated dissipation of $\Delta \mathrm{pH}$ in starved cells of strains Doc-S and TUV, as monitored by NMR spectroscopy (Fig. 3), provides direct evidence that TTFB has equal and facile access to the cytoplasmic membranes of both, and argues against any reduction in the envelope permeability of strain TUV under these conditions. The fact that $\Delta \mathrm{pH}$ is dissipated at similar rates in both strains also argues against any decrease in proton transport rates within the cytoplasmic membrane of strain TUV, implying that uncoupler function within this membrane is unimpaired. Furthermore, the pH dependence of TTFB's uncoupling activity in strains Doc-S and TUV is identical to that of its protonophoric activity in phospholipid bilayers (Dilger \& McLaughlin, 1979), arguing against the involvement of bacterial membrane proteins in the uncoupling process. Dissipation of $\Delta \mathrm{pH}$, however, was always slow $(\leq 0.05 \mathrm{pH}$ units $\min ^{-1}$ ), raising the possibility that proton translocation is not rate-limiting in the uncoupling process. For example, FCCP dissipates the mitochondrial $\Delta p$ within seconds (Bakker et al., 1973), but $\Delta \mathrm{pH}$ collapses slowly, possibly because of slow counterion movements (Ogawa et al., 1981). If similar processes limit the rate of collapse of $\Delta \mathrm{pH}$ in $E$. coli, then any impairment of TTFB's protonophoric activity in strain TUV might remain undetected.

The evidence discussed so far may be interpreted in favour of a model in which uncoupler resistance is maintained by active, substrate-dependent processes, with retention of $\Delta \mathrm{pH}$ (in agreement with Ito et al., 1983; Sedgwick et al., 1984). A possible origin of the active resistance of strain TUV lies in its $25 \%$-enhanced respiratory rate, which could promote faster proton translocation and greater protonophore resistance. The cytochromes of strains Doc-S and TUV appear qualitatively and quantitatively indistinguishable (data not shown), suggesting an unchanged $\mathrm{H}^{+} / 2 \mathrm{e}^{-}$stoichiometry; thus proton translocation in strain TUV is unlikely to increase by more than $25 \%$. Such a modest increase seems insufficient to explain the strain's 10fold enhanced TTFB resistance (Table 1), unless other factors are also involved.

Evidence that the situation is more complicated than was at first thought comes from the finding of novobiocin resistance in strains TUV and CUV, suggesting a reduced envelope permeability in these strains. Preliminary genetic analysis (by $\mathrm{O}$. Michelsen, University of Odense, Denmark) indicates that strain Doc-S carries a mutation conferring resistance to phage P1 and cotransducible with the pyrE locus, which has reverted to wild-type in strain TUV. A likely location of the change is the $r f a$ operon. This operon encodes several enzymes concerned with lipopolysaccharide synthesis (Nikaido \& Vaara, 1985), and rfa mutant strains can be extremely sensitive to novobiocin. (This cannot be the only lesion affecting the envelope of strain Doc-S and its derivatives, since strains TUV and CUV retain abnormal sensitivity to deoxycholate.) It is not yet known whether strain TUV carries further mutations from its parent (strain CUV, presumably, must), but a $r f a$ back-mutation could account for the reduction in novobiocin sensitivity, and might lead to a more effective exclusion of TTFB and FCCP. Such a 
mutation could also account for the cross-resistance pattern of strain TUV, including the retention of sensitivity to DNP, a hydrophilic uncoupler which could still reach the cell membrane through the pores of the outer wall. Interestingly, strain UV6 also retained sensitivity to DNP (Sedgwick et al., 1984). Furthermore, the rfa operon lies at $81 \mathrm{~min}$ on the chromosome, quite close to the unc operon $(83.5 \mathrm{~min})$, raising the possibility that some of the mutations documented by Ito and co-workers (Ito \& Ohnishi, 1981, 1982; Ito et al., 1983) originated in the $r f a$ region. The reduced permeability of strain TUV to TPP ${ }^{+}$is further evidence of a cell envelope change.

Although the above findings might appear to lead to an essentially trivial explanation of uncoupler resistance in these strains, two interesting questions remain. Firstly, one would expect resistance arising from a cell envelope mutation to be passive, whereas the resistance of strain TUV contains an essential active component. Secondly, the uncoupler resistance of strains TUV and CUV is much greater than that of strains possessing wild-type envelopes, even though the former strains retain abnormal sensitivity to deoxycholate. A possible solution to the first problem comes from a report that cellular energization may render the wild-type $E$. coli envelope less permeable to uncouplers (Helgerson \& Cramer, 1977). The nature of cell membrane-cell wall interactions in E. coli remains obscure, but proteins spanning the periplasm have recently been described, which may be involved in the energy-dependent opening and closing of cell wall pores (Higgins et al., 1988). Thus, the access of uncouplers to the cell membrane of strain TUV may become impaired by changes in cell wall permeability resulting from cellular energization. A partial recovery of envelope integrity might also explain the enhanced growth and respiratory rates of the resistant strains.

In conclusion, it appears that complex cell membrane-cell wall interactions may occur even in deoxycholate-sensitive strains of $E$. coli, and that these might be modulated by the degree of cellular energization. Our results are generally compatible with previous studies of uncoupler resistance in $E$. coli, but the diverse properties of the various mutant strains may not all be explicable in terms of a single underlying mechanism. We would, however, draw a distinction between results obtained with $E$. coli and those obtained with resistant strains of the Grampositive organisms Bacillus megaterium and B. subtilis. In the latter species, ATP synthesis was maintained despite the unimpaired dissipation of $\Delta p$ by uncouplers, and resistance correlated with changes in membrane lipids (Krulwich et al., 1987; Clejan et al., 1988). While the biochemistry of uncoupler resistance in $E$. coli remains intriguing, the type of mutation exemplified by the Bacillus species may be more challenging from a bioenergetic standpoint.

We thank N. Soffe and Dr J. Boyd for their help with the NMR spectrometer, and Dr O. Michelsen for performing the mapping studies. We thank SERC, the Wellcome Trust and the Nuffield Foundation for financial support, and Dr A. A. Guffanti for helpful discussions.

\section{REFERENCES}

AHMED, S. \& BoOTH, I. R. (1983). The use of valinomycin, nigericin and trichlorocarbanilide in control of the protonmotive force in Escherichia coli cells. Biochemical Journal 212, 105-112.

Bakker, E. P., van den Heuvel, E. J., Wiechmann, A. H. C. A. \& VAN DAM, K. (1973). A comparison between the effectiveness of uncouplers of oxidative phosphorylation in mitochondria and in different artificial membrane systems. Biochimica et biophy. sica acta 292, 78-87.

Burstein, C., Tiankova, L. \& KePes, A. (1979). Respiratory control in Escherichia coli K12. European Journal of Biochemistry 94, 387-392.

Clejan, S., Guffanti, A. A., Falk, L. H. \& KRULwich, T. A. (1988). The protonophore resistance of Bacillus subtilis is correlated with elevated ratios of saturated to unsaturated fatty acids in membrane phospholipids. Biochimica et biophysica acta 932, 43-51.

Cozzarelli, N. R. (1980). DNA gyrase and the supercoiling of DNA. Science 207, 953-960.

DATE, T., ZWIZINSKI, C., LUDMERER, S. \& WiCKNER, W. (1980). Mechanisms of membrane assembly: effects of energy poisons on the conversion of soluble M13 coliphage procoat to membrane-bound coat protein. Proceedings of the National Academy of Sciences of the United States of America 77, 827-831.

Dilger, J. \& Mclaughlin, S. (1979). Proton transport through membranes induced by weak acids: a study of two substituted benzimidazoles. Journal of Membrane Biology 46, 359-384.

DugGleBY, R. G. (1984). Regression analysis of nonlinear Arrhenius plots: an empirical model and 
computer program. Computers in Biology and Medicine 14, 447-455.

Gutowski, S. J. \& Rosenberg, H. (1975). Succinate uptake and related proton movements in Escherichia coli K12. Biochemical Journal 152, 647-654.

Helgerson, S. L. \& Cramer, W. A. (1977). Changes in Escherichia coli cell envelope structure and the sites of fluorescence probe binding caused by carbonyl cyanide $p$-trifluoromethoxyphenylhydrazone. Biochemistry 16, 4109-4117.

Higgins, C. F., Barr, G. C., Adamson, J. \& Dorman, C. J. (1988). The TonB protein: a mechanism for coupling energy to the bacterial outer membrane. 5th EBEC Conference Short Reports, p. 15.

ITO, M. \& OHNISHI, Y. (1981). Isolation of Escherichia coli mutants which are resistant to an inhibitor of $\mathrm{H}^{+}$-ATPase, tributyltin and also to uncouplers of oxidative phosphorylation. FEBS Letters 136, 225230.

ITO, M. \& OHNISHI, Y. (1982). Escherichia coli mutants resistant to uncouplers of oxidative phosphorylation. Microbiology and Immunology 26, 1079-1084.

Ito, M., OHNISHI, Y., IтOH, S. \& NishimURA, M. (1983). Carbonyl cyanide- $m$-chlorophenyl hydrazoneresistant Escherichia coli mutant that exhibits a temperature-sensitive Unc phenotype. Journal of Bacteriology 153, 310-315.

JONES, M. R. \& BEECHEY, R. B. (1987). Escherichia coli mutants resistant to uncouplers of oxidative phosphorylation. Journal of General Microbiology 133, 2759-2766.

Jones, M. R., Quirk, P. G., CAMPbell, I. D. \& BEECHEY, R. B. (1986). Uncoupler-resistance in Escherichia coli: the effect of uncouplers on proline uptake, ATP levels and the transmembrane $\mathrm{pH}$ gradient. Biochemical Society Transactions 14, 888889.

Kagawa, Y., Sone, N., Hirata, H. \& Yoshida, M. (1979). Structure and function of $\mathrm{H}^{+}$-ATPase. Journal of Bioenergetics and Biomembranes 11, 39-78.

Katre, N. V. \& Wilson, D. F. (1980). A specific uncoupler-binding protein in Tetrahymena pyriformis and Paracoccus denitrificans. Biochimica et biophysica acta 593, 224-229.

Kell, D. B. (1982). Bacteria that are resistant to uncouplers - what can they tell us? Trends in Biochemical Sciences 7, 1-2.

Krulwich, T. A., Clejan, S., Falk, L. H. \& GuFFANTI, A. A. (1987). Incorporation of specific exogenous fatty acids into membrane lipids modulates protonophore resistance in Bacillus subtilis. Journal of Bacteriology 169, 4479-4485.

Mclaughlin, S. G. A. \& Dilger, J. P. (1980). Transport of protons across membranes by weak acids. Physiological Reviews 60, 825-863.

Mrtchell, P. (1961). Coupling of phosphorylation to electron and hydrogen transfer by a chemiosmotic type of mechanism. Nature, London 191, 144-148.

NikAIDO, H. \& VAARA, M. (1985). Molecular basis of bacterial outer membrane permeability. Microbiological Reviews 49, 1-32.

Ogawa, S., Shulman, R. G., Glynn, P., Yamane, T. \& Navon, G. (1978). On the measurement of pH in Escherichia coli by ${ }^{31} \mathrm{P}$ nuclear magnetic resonance. Biochimica et biophysica acta 502, 45-50.

Ogawa, S., Boens, C. C. \& LeE, T.-M. (1981). A ${ }^{31}$ P nuclear magnetic resonance study of the $\mathrm{pH}$ gradient and the inorganic phosphate distribution across the membrane in intact rat liver mitochondria. Archives of Biochemistry and Biophysics 210, 740-747.

Partis, M. D., Griffiths, D. G. \& BeEchey, R. B. (1984). Discrimination between the binding sites of modulators of the $\mathrm{H}^{+}$-translocating ATPase activity in rat liver mitochondrial membranes. Archives of Biochemistry and Biophysics 232, 610-615.

SedGwick, E. G., Hou, C. \& BragG, P. D. (1984). Effect of uncouplers on the bioenergetic properties of a carbonyl cyanide- $m$-chlorophenylhydrazoneresistant mutant Escherichia coli UV6. Biochimica et biophysica acta 767, 479-492.

Selwyn, M. J., Dawson, A. P., Stockdale, M. \& GaINs, N. (1970). Chloride-hydroxide exchange across mitochondrial, erythrocyte and artificial lipid membranes mediated by trialkyl- and triphenyltin compounds. European Journal of Biochemistry 14, $120-126$.

Slonczewski, J. L., Rosen, B. P., Alger, J. R. \& MACNAB, R. M. (1981). pH homeostasis in Escherichia coli: measurement by ${ }^{31} \mathrm{P}$ nuclear magnetic resonance of methylphosphonate and phosphate. Proceedings of the National Academy of Sciences of the United States of America 78, 6271-6275.

Ugurbil, K., RotTenberg, H., GlynN, P. \& ShulMAN, R. G. (1978). ${ }^{31} \mathrm{P}$ nuclear magnetic resonance studies of bioenergetics and glycolysis in anaerobic Escherichia coli cells. Proceedings of the National Academy of Sciences of the United States of America 75, 2244-2248. 